\title{
Measurement of Fruit and Vegetable Intake Incorporating a Diversity, Equity, and Inclusion Lens. Comment on Di Noia, J.; Gellermann, W. Use of the Spectroscopy-Based Veggie Meter ${ }^{\circledR}$ to Objectively Assess Fruit and Vegetable Intake in Low-Income Adults. Nutrients 2021, 13, 2270
}

\author{
Carmen Byker Shanks ${ }^{1,2, * \mathbb{D}}$, Betty Izumi ${ }^{3}$, Courtney A. Parks ${ }^{1}$ and Amy L. Yaroch ${ }^{1}$ \\ 1 Gretchen Swanson Center for Nutrition, Omaha, NE 58154, USA; cparks@centerfornutrition.org (C.A.P.); \\ ayaroch@centerfornutrition.org (A.L.Y.) \\ 2 Department of Health and Human Development, Montana State University, Bozeman, MT 59717, USA \\ 3 OHSU-PSU School of Public Health, Oregon Health and Science University-Portland State University, \\ Portland, OR 97201, USA; izumibet@pdx.edu \\ * Correspondence: cbshanks@centerfornutrition.org; Tel.: +1-(531)-895-4037
}

Citation: Byker Shanks, C.; Izumi, B.; Parks, C.A.; Yaroch, A.L.

Measurement of Fruit and Vegetable Intake Incorporating a Diversity,

Equity, and Inclusion Lens. Comment on Di Noia, J.; Gellermann, W. Use of the Spectroscopy-Based Veggie Meter $^{\circledR}$ to Objectively Assess Fruit and Vegetable Intake in Low-Income Adults. Nutrients 2021, 13, 2270. Nutrients 2022, 14, 809. https:// doi.org/10.3390/nu14040809

Academic Editor: Gunter G. C. Kuhnle

Received: 19 July 2021

Accepted: 7 February 2022

Published: 15 February 2022

Publisher's Note: MDPI stays neutral with regard to jurisdictional claims in published maps and institutional affiliations.

Copyright: (C) 2022 by the authors. Licensee MDPI, Basel, Switzerland. This article is an open access article distributed under the terms and conditions of the Creative Commons Attribution (CC BY) license (https:// creativecommons.org/licenses/by/ $4.0 /)$.
Disparities in fruit and vegetable intake (FVI) and diet-related diseases exist among lowincome and racial/ethnic minority populations [1-4]. Intervention approaches to eliminate FVI disparities frequently utilize dietary assessment to measure impact. Studies measure FVI in varying ways, but do not fully account for diversity, equity, and inclusion (DEI).

Di Noia and Gellerman upheld the Veggie Meter using resonance Raman spectrometry as a non-invasive, objective biomarker approach to measure FVI for assessing dermal carotenoids among low-income populations [5]. However, a meta-analysis reported that the validity of resonance Raman spectrometry has mainly been established among White populations, and research among non-White populations is warranted [6].

Dietary recalls assess FVI by capturing a variety of self-reported foods [7]. Recall data are entered into food analysis software, and these data are limited by the foods available in the database. Researchers find substitute foods when the participant's response is not in the database. For example, the fruit atemoya is consumed across cultures, but not found in most databases.

Screeners are frequently employed in intervention studies that target FVI, since they are brief, inexpensive, and assess defined variables of interest [8]. Screeners are limited by items assessed within a food group (e.g., tomato sauce represents the red/orange vegetable subgroup) or example foods (e.g., delimiting labeling such as "Mexican-type salsa with tomato").

FVI is an indicator of a behavior and, if interpreted out of context, can be used to place blame on the individual for poor dietary choices. Pairing FVI with other known data sources (e.g., food access) can shift the conversation to focus on systematic and external barriers that influence dietary patterns among populations.

Sociodemographic categories also may not reflect actual identities. In the U.S., FVI measures are typically validated in English, and should be expanded to other languages to ensure the relevance of translations. Questions based upon sex/gender customarily ask about the female and male genders, and do not extend to non-binary, third gender, and/or self-described options. Racial/ethnic groups are typically limited to five categories specified in the 1997 Office of Management and Budget (OMB) standards on race and ethnicity, and do not address the heterogeneity among groups in responsive and inclusive ways [9].

FVI and related data are potentially powerful tools to illuminate systemic inequities, but they are only as useful as their applicability across populations. Measures integrat- 
ing diversity (the ways in which individuals differ) could ensure the inclusion of the variety/types of FVs consumed to reflect the intake of diverse populations. Measures integrating equity (equal access to opportunities) could measure factors that drive FVI. Measures integrating inclusion (including all individuals) could address sociodemographic variables and languages with which people identify. The ability to understand the effectiveness of interventions in eliminating disparities in FVI is dependent upon measures adequately representing dietary patterns and influencing factors, but this is not feasible without incorporating DEI.

Funding: This work was supported by Gus Schumacher Nutrition Incentive Grant Program grant no. 2019-70030-30415/project accession no. 1020863 from the United States Department of Agriculture (USDA) National Institute of Food and Agriculture and an Institutional Development Award (IDeA) from the National Institute of General Medical Sciences of the National Institutes of Health (NIH) under grant number P20GM104417. The content is solely the responsibility of the authors and does not necessarily represent the official views of the USDA and NIH.

Conflicts of Interest: The authors declare no conflict of interest.

\section{References}

1. Martin, C.; Hoy, M.K.; Clemens, J.; Moshfegh, A. Demographic characteristics associated with variety of fruit and vegetable intake: What we eat in America, NHANES 2013-2016 (FS02-06-19). Curr. Dev. Nutr. 2019, 3, nzz051-FS02-06-19. [CrossRef]

2. Gearing, M.; Lewis, M.; Wilson, C.; Bozzolo, C.; Hansen, D. Barriers That Constrain the Adequacy of Supplemental Nutrition Assistance Program (SNAP) Allotments: In-Depth Interview Findings; Report prepared by Westat, Inc. for the U.S. Department of Agriculture, Food and Nutrition; Project Officer Rosemarie Downer; Westat, Inc.: Alexandria, VA, USA, 2021. Available online: www.fns.usda.gov / research-and-analysis (accessed on 10 February 2022).

3. Hales, C.M.; Carroll, M.D.; Fryar, C.D.; Ogden, C.L. Prevalence of Obesity among Adults and Youth: United States, 2015-2016. NCHS Data Brief, No 288; National Center for Health Statistics: Hyattsville, MD, USA, 2017.

4. Hill-Briggs, F.; Adler, N.E.; Berkowitz, S.A.; Chin, M.H.; Gary-Webb, T.L.; Navas-Acien, A.; Thornton, P.L.; Haire-Joshu, D. Social determinants of health and diabetes: A scientific review. Diabetes Care 2021, 44, 258-279. [CrossRef] [PubMed]

5. Di Noia, J.; Gellermann, W. Use of the spectroscopy-based Veggie Meter ${ }^{\circledR}$ to objectively assess fruit and vegetable intake in low-income adults. Nutrients 2021, 13, 2270. [CrossRef] [PubMed]

6. Jilcott Pitts, S.B.; Johnson, N.S.; Wu, Q.; Firnhaber, G.C.; Preet Kaur, A.; Obasohan, J. A meta-analysis of studies examining associations between resonance Raman spectroscopy-assessed skin carotenoids and plasma carotenoids among adults and children. Nutr. Rev. 2021, 80, 230-241. [CrossRef] [PubMed]

7. National Cancer Institute. 24-hour Dietary Recall (24HR) at a Glance. Available online: https:/ / dietassessmentprimer.cancer. gov / profiles/recall/ (accessed on 19 July 2021).

8. National Cancer Institute. Screeners at a Glance. Available online: https://dietassessmentprimer.cancer.gov/profiles/screeners/ index.html (accessed on 19 July 2021).

9. United States Census. Research to Improve Data on Race and Ethnicity. Available online: https://www.census.gov/about/ourresearch/race-ethnicity.html (accessed on 19 July 2021). 